\title{
Ciclo de Mejora en el Aula mediante Aprendizaje Basado en Problemas en la asignatura práctica Nutrición y Bromatología
}

\section{Applying investigative logic in an improvement cycle in classroom in the practical subject Nutrition and Bromatology}

PAUlA MAPELLI BRAHM

ORCID: https://orcid.org/0000-0003-1940-442X

Universidad de Sevilla

Departamento de Nutrición y Bromatología, Toxicología y Medicina Legal

pmapelli@us.es

DOI: http://dx.doi.org/10.12795/9788447231003.044

Pp.: 925-946 


\section{Introducción y contexto}

El Ciclo de Mejora en el Aula (CIMA) se ha llevado a cabo con doce alumnos del 3 o curso del grado de farmacia, en las prácticas de Nutrición y Bromatología. Estas prácticas forman parte de la asignatura cuatrimestral obligatoria del mismo nombre, que se imparte desde el departamento de Nutrición y Bromatología, Toxicología y Medicina Legal de la Universidad de Sevilla. Las prácticas de laboratorio son de asistencia obligatoria y deben aprobarse para poder superar la asignatura completa.

Dadas las circunstancias excepcionales que vivimos estos días cómo consecuencia de la pandemia de enfermedad por coronavirus, la mitad de las clases de esta asignatura se impartieron en el laboratorio de forma presencial y la otra mitad de forma virtual.

\section{Diseño previo del CIMA}

\section{Mapa de contenidos y problemas claves}

La temática de esta asignatura práctica está relacionada con el análisis de ciertos alimentos y nutrientes. En mi opinión, el análisis de alimentos es un tema que puede resultar interesante para los alumnos, ya que, en general, la sociedad actual está cada vez más preocupada por temas relacionados con la alimentación. En particular, se trabajarán las siguientes prácticas de laboratorio: el análisis del color, las cenizas, proteínas, grasas y el almidón en productos cárnicos, la determinación de cafeína en ciertas bebidas y el análisis de la humedad en mieles (Figura 1).

Ciclos de Mejora en el Aula (2020). Experiencias de Innovación Docente de la US Esta obra se distribuye con la licencia Creative Commons 
Como puede observarse en la Figura 1, los problemas claves a tratar son ¿para qué y cómo analizamos los alimentos? En relación al ¿cómo analizamos los alimentos?, en este CIMA se tratarán desde un punto de vista teórico (contenidos conceptuales, sin parte práctica), además de los métodos de análisis de referencia para los alumnos (Figura 1), nuevos métodos no contemplados con anterioridad. Estos nuevos métodos analíticos surgirán a través de una serie de actividades en las que el docente no dará la información de forma directa, sino que serán los propios estudiantes los que buscarán la información a aprender. De esta forma, los contenidos aprendidos dependerán de los resultados del trabajo realizado (Cubero y Carmona, 1994). Incluyendo nuevas metodologías analíticas en el contenido de la asignatura se pretende que los alumnos puedan tener una visión más amplia del análisis de alimentos y dispongan de información suficiente como para poder comparar unos métodos con otros y razonar sobre los mismos. Tal como se aprecia en la Figura 1, en esta pregunta (¿Cómo?) se incluyen contenidos procedimentales como son la realización de la práctica correspondiente (análisis del compuesto en el laboratorio siguiendo la metodología de referencia), la resolución de problemas que puedan surgir durante el análisis, y la realización de ejercicios de cálculos para obtener los resultados que se buscan.

Ciclos de Mejora en el Aula (2020). Experiencias de Innovación Docente de la US Esta obra se distribuye con la licencia Creative Commons 


\section{PAUla MAPELLI BRAHM}

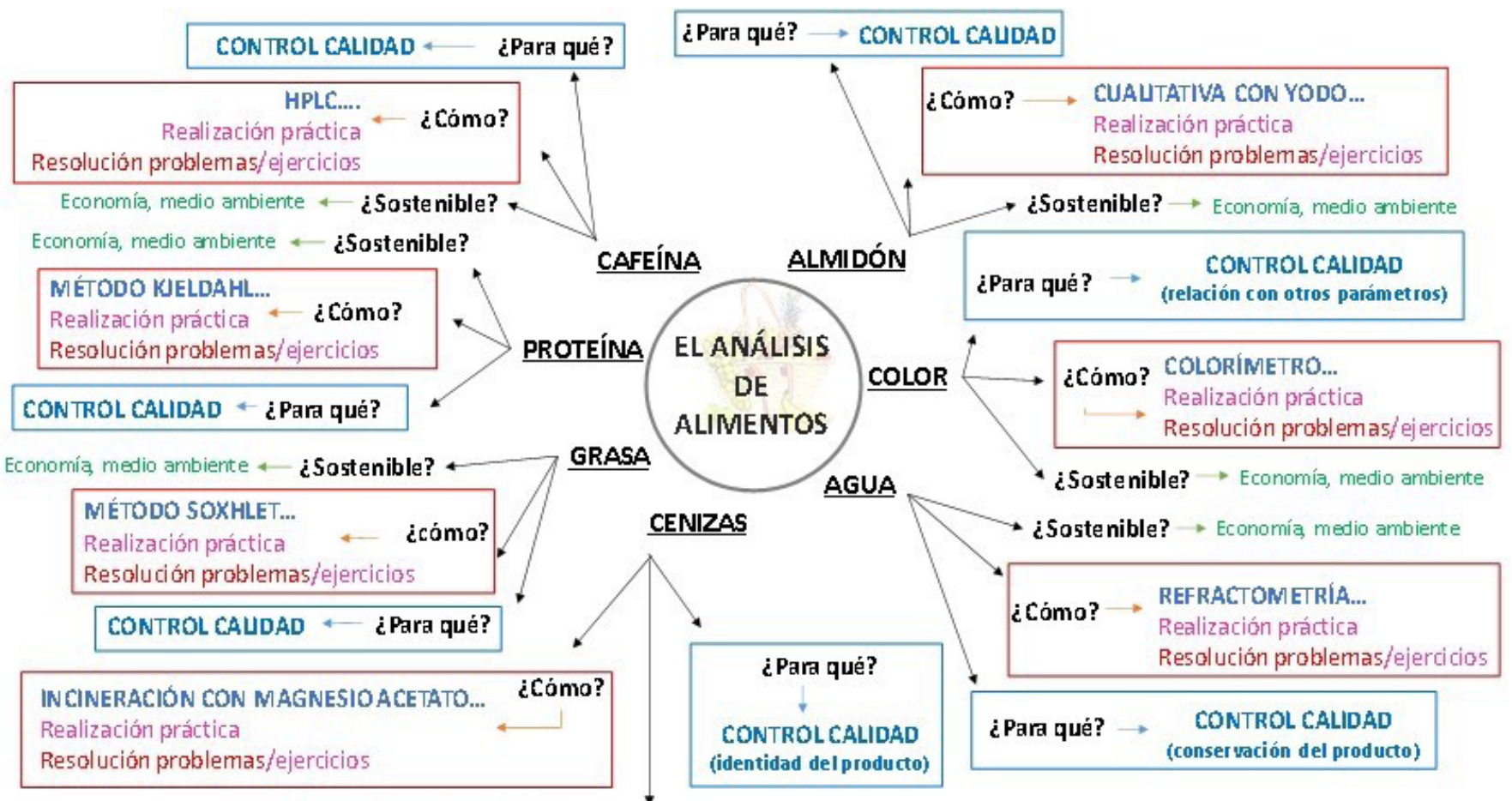

Economía, medio ambiente 4 ¿Sostenible?

CONTENIDOS ESBNCIALES: BN RECUADROS

Figura 1. Mapa de Contenidos

Ciclos de Mejora en el Aula (2020). Experiencias de Innovación Docente de la US

cc (i) E $\Theta$ Esta obra se distribuye con la licencia Creative Commons 
Los compuestos objeto de interés en la asignatura aparecen subrayados. De cada compuesto se tienen tres preguntas relacionadas con la metodología de análisis de los mismos. Los problemas claves se encuentran en los recuadros (¿Cómo y para qué analizamos los alimentos?). En mayúscula en el interior del recuadro rojo aparecen los métodos de referencia para los alumnos (la información detallada de los mismos está disponible en el cuadernillo de prácticas del que disponen los alumnos). Tipos de contenidos: Procedimentales psicomotrices (en rosa), procedimentales intelectuales (en rojo), actitudinales (en verde) y conceptuales (en azul)

Por otro lado, se ha incluido como problema clave el ¿para qué analizamos los alimentos?, por ser esta una pregunta fundamental para dar sentido a las prácticas y motivar así a los estudiantes. Este ha sido uno de los cambios realizados con el CIMA, pues en años anteriores me he enfocado fundamentalmente en cómo analizamos los alimentos.

Porlán (2017) indica que a la hora de diseñar los contenidos de enseñanza se deben incluir, además de los contenidos conceptuales (saber) y procedimentales (saber hacer), contenidos actitudinales (saber ser) que incluyen actitudes, valores, normas y posicionamientos éticos. Atendiendo a este razonamiento, en este CIMA se han incluido nuevos contenidos de tipo actitudinales no planteados en esta asignatura en años anteriores (Figura 1). Así, para cada método de análisis estudiado, se evaluará el impacto económico del mismo y si este es o no respetuoso con el medio ambiente. Se pretende así que los alumnos razonen sobre ciertos aspectos importantes en la actualidad para la sociedad relacionados con los contenidos planteados en esta asignatura.

Ciclos de Mejora en el Aula (2020). Experiencias de Innovación Docente de la US Esta obra se distribuye con la licencia Creative Commons 


\section{Modelo metodológico posible}

En la Figura 2 se muestra un resumen de la metodología a usar para desarrollar este CIMA y de la metodología habitual usada en años anteriores. Uno de los principales problemas de la metodología habitual es que aquellos alumnos que están poco motivados, realizan la práctica sin entender qué están haciendo ni para qué lo están haciendo, pero obtienen el mismo resultado que el resto de compañeros, pues leyendo el cuadernillo de prácticas son capaces de realizarlas como si de una receta de cocina se tratase. Por ello, a través del presente CIMA se pretende mejorar la metodología que se usa normalmente para impartir esta asignatura, incentivar el interés de los estudiantes y obtener mejores resultados en el proceso de enseñanza-aprendizaje.

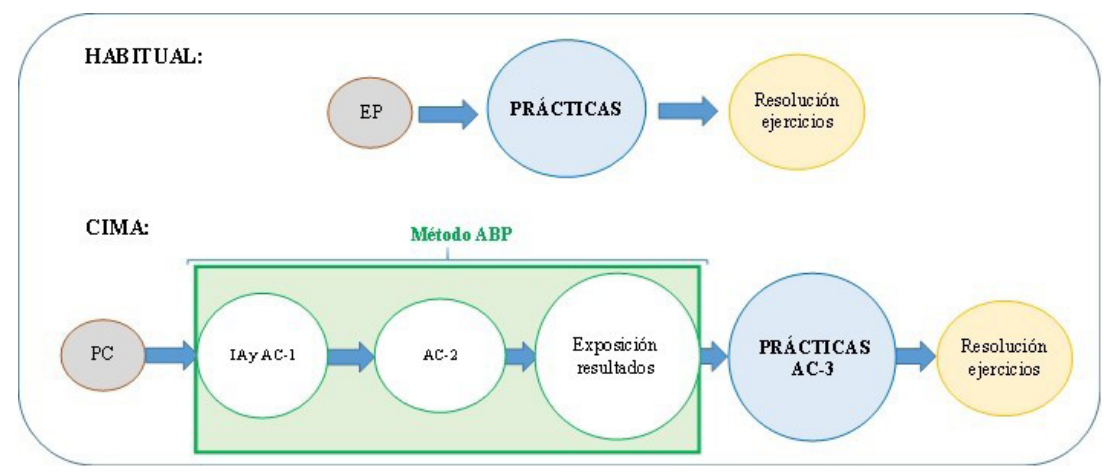

Figura 2. Resumen de la metodología habitual y la metodología del CIMA.

EP, Explicación de la Prácticas; ABP, Aprendizaje Basado en Proyectos; PC; Problemas Clave; IA, Ideas de los Alumnos; AC-1, -2 y -3, Actividad de Contraste -1, -2 y -3. El tamaño de las circunferencias hace referencia a la importancia que tiene cada elemento en el modelo metodológico

Como puede observarse en la Figura 2, el modelo actual surge de unos problemas claves que el docente presentará a los alumnos. Como ya se indicó anteriormente,

Ciclos de Mejora en el Aula (2020). Experiencias de Innovación Docente de la US Esta obra se distribuye con la licencia Creative Commons 
los problemas claves a tratar son ¿para qué y cómo analizamos los alimentos?

Para resolver estos problemas se trabajará en grupos con el método de Aprendizaje Basado en Problemas (ABP). Este método incluirá una primera fase en la que los alumnos responderán a los problemas claves sin buscar información (Idea de los Alumnos, IA), para después continuar con la búsqueda de información con el fin de seguir elaborando las respuestas (Actividad de Contraste 1, AC-1). En una segunda fase, el docente entrará en acción, guiando la investigación y la reflexión de los estudiantes a través de la incorporación de nuevas preguntas (AC-2). La última fase consistirá en la exposición por parte de los estudiantes de los resultados de la investigación. Los problemas claves a tratar en las actividades del método ABP serán preguntas abiertas; el docente no indicará qué métodos de análisis son los que tienen que investigar, sino que dejará que sean ellos los que busquen las diferentes opciones a evaluar y comparar. Se busca así un aprendizaje por descubrimiento, en el que no se aporta de forma directa la información que los estudiantes tienen que asimilar (Cubero y Carmona, 1994). Se pretende de esta forma captar el interés de los estudiantes y que, desde el trabajo del propio alumno, surja un conocimiento profundo y duradero. En este modelo, el docente, al contrario que en el modelo habitual, tiene un papel diferente en el proceso de enseñanza-aprendizaje, actuando simplemente como guía y orientador en este proceso.

Al tratarse de una asignatura práctica, el modelo actual, al igual que el habitual, incluirá la realización de una serie de prácticas. La diferencia es que en este caso se plantearán como una actividad de contraste (AC-3). Es decir, a través de la realización de las prácticas los estudiantes podrán contrastar lo aprendido a través del método ABP.

Ciclos de Mejora en el Aula (2020). Experiencias de Innovación Docente de la US Esta obra se distribuye con la licencia Creative Commons 
Durante la resolución de los ejercicios de cálculo (necesarios para conseguir, a partir de los datos obtenidos durante las prácticas, los resultados que se buscan), el docente seguirá actuando de guía, siendo los alumnos los que de forma autónoma deberán tratar de resolver los ejercicios.

Como resumen, en este proyecto se trabajarán con diversas estrategias metodológicas (trabajo en grupo, trabajo escrito, exposición y prácticas), procurando que las clases sean bastante dinámicas, que el alumnado sea activo y que la comunicación con el profesor sea bidireccional. El proyecto fomentará la curiosidad y se basará en el autoaprendizaje con el fin de que los alumnos mejoren su capacidad de "aprender a aprender".

\section{Secuencia de actividades programadas}

Este modelo metodológico se traduce en una serie de actividades que se desarrollarán en tres sesiones diferentes (9 h en total) (Tabla 1). Estas actividades han sido diseñadas con el fin de conseguir un aprendizaje efectivo aplicando los consejos de Bain (2007). Así, los estudiantes tendrán que resolver problemas que consideran atractivos e importantes (problemas claves), trabajarán con el apoyo del docente y de otros alumnos para superar problemas, sus trabajos serán valorados justamente y recibirán retroalimentación de otros estudiantes. Las actividades 3-6 (Tabla 1) representan una parte importante de la secuencia de actividades y están basadas en el método ABP. Este conjunto de actividades (3-6) se ha diseñado para ser una experiencia de aprendizaje sostenida para los alumnos. Para ello, se ha trazado una secuencia lógica con un principio motivador (Actividad 3), pasos intermedios (Actividades 4-5) y un desenlace final (Actividad 6) (Finkel, 2008).

Ciclos de Mejora en el Aula (2020). Experiencias de Innovación Docente de la US Esta obra se distribuye con la licencia Creative Commons 
Tabla 1. Secuencias de actividades programas.

\begin{tabular}{|c|c|c|}
\hline \multicolumn{3}{|c|}{ SESIÓN 1} \\
\hline Act. 1 & \multirow{2}{*}{ PRESENTACIÓN } & PC \\
\hline $15 \mathrm{~min}$ & & Foro E.V. \\
\hline \multicolumn{3}{|c|}{$\begin{array}{l}\text { Consiste en la presentación y justificación de los problemas centrales } \\
\text { (¿cómo y para qué analizamos los alimentos?). Se explicará cuáles son los } \\
\text { principales contenidos a tratar y el contexto imaginario en el que se van a } \\
\text { situar (Método ABP (actividades 3-6)). }\end{array}$} \\
\hline Act. 2 & \multirow{2}{*}{ CUESTIONARIO INICIAL } & $\mathrm{Cl}$ \\
\hline $30 \mathrm{~min}$ & & Google Docs \\
\hline \multicolumn{3}{|c|}{$\begin{array}{l}\text { Se les pasará a los alumnos un cuestionario de ideas previas. Se hará } \\
\text { hincapié en el hecho de que este no tendrá repercusión en la nota final de } \\
\text { la asignatura y que será anónimo. }\end{array}$} \\
\hline Act. 3 & \multirow{2}{*}{$\begin{array}{c}\text { IDEAS DE LOS ALUMNOS SOBRE CIERTAS } \\
\text { PREGUNTAS }\end{array}$} & IA \\
\hline $15 \mathrm{~min}$ & & $\begin{array}{c}\text { Trabajos en grupo en } \\
\text { E.V. }\end{array}$ \\
\hline \multicolumn{3}{|c|}{$\begin{array}{l}\text { El profesor explicará en qué consiste la tarea: responder en parejas (6 } \\
\text { parejas en total), sin buscar información, a tres preguntas similares a las del } \\
\text { cuestionario inicial, pero adaptadas a cada compuesto y matriz alimentaria. } \\
\text { El profesor permitiá que los alumnos creen los grupos y ayudará a que } \\
\text { estos se formen con la mayor celeridad posible. Cada pareja trabajará } \\
\text { con un compuesto y una matriz alimentaria y lo hará en una de las salas } \\
\text { creadas por el docente en la plataforma de la E.V. El profesor pasará por las } \\
\text { diferentes salas para comprobar que los alumnos están trabajando y para } \\
\text { resolver posibles dudas. } \\
\text { Las respuestas deben quedar reflejadas en un informe que deberán } \\
\text { entregar al final de la sesión } 1 \text {. En este informe deberán indicar los posibles } \\
\text { acuerdos/desacuerdos encontrados. }\end{array}$} \\
\hline Act. 4 & \multirow{2}{*}{$\begin{array}{l}\text { BÚSQUEDA INFORMACIÓN PARA } \\
\text { RESPONDER A LAS PREGUNTAS }\end{array}$} & $A C-1$ \\
\hline $1 \mathrm{~h}$ & & $\begin{array}{c}\text { Trabajos en grupo en } \\
\text { E.V. }\end{array}$ \\
\hline \multicolumn{3}{|c|}{$\begin{array}{l}\text { A continuación, se les informará a los alumnos que pueden continuar con } \\
\text { el desarrollo de las respuestas, pero buscando información. El docente } \\
\text { indicará ciertas pautas a seguir para la búsqueda de información y pasará } \\
\text { por las diferentes salas para comprobar que las fuentes donde buscan } \\
\text { la información sean aceptables, para resolver dudas, para dar algunos } \\
\text { consejos, etc. }\end{array}$} \\
\hline
\end{tabular}

Ciclos de Mejora en el Aula (2020). Experiencias de Innovación Docente de la US Esta obra se distribuye con la licencia Creative Commons Reconocimiento-NoComercial-SinObraDerivada Internacional (CC BY-NC-ND 4.0.) 


\begin{tabular}{|c|c|c|}
\hline Act. 5 & \multirow{2}{*}{$\begin{array}{c}\text { GUÍA EN LAS RESPUESTAS A LAS } \\
\text { PREGUNTAS }\end{array}$} & $\begin{array}{c}\text { Trabajos en grupo en } \\
\text { E.V. }\end{array}$ \\
\hline
\end{tabular}

El profesor realizará nuevas preguntas para guiar y reconducir la investigación y la reflexión de los alumnos. Por ejemplo: ¿habéis pensado en la sostenibilidad del método? ¿es un método destructivo, es sencillo de realizar? Además, irá realizando las correcciones oportunas sobre los trabajos.

Al finalizar la sesión 1, los alumnos deberán entregar una presentación con los resultados finales del trabajo. Las respuestas finales a las preguntas deberán incluirse en el informe a entregar (junto con las de la Act. 3). El profesor revisará los trabajos e enviará a los alumnos antes de la siguiente sesión (al día siguiente) algunos comentarios, correcciones, etc.

\begin{tabular}{|c|c|c|}
\hline \multicolumn{3}{|c|}{ SESIÓN 2} \\
\hline Act. 6 & \multirow{2}{*}{ EXPOSICIÓN DE LOS RESULTADOS } & ER \\
\hline $2 \mathrm{~h}$ & & PowerPoint en E.V. \\
\hline \multicolumn{3}{|c|}{$\begin{array}{l}\text { Los alumnos deberán exponer (10 min) en parejas al resto de compañeros } \\
\text { el trabajo realizado. A continuación, se abrirá un turno de preguntas. } \\
\text { El docente realizará las puntualizaciones/correcciones necesarias y } \\
\text { actuará de guía en los posibles debates. El tiempo de dudas/debate/ } \\
\text { puntualizaciones será de aproximadamente } 10 \text { min. Con esta actividad } \\
\text { se pretende que los alumnos aprendan de sus compañeros y mejoren } \\
\text { su capacidad de expresión oral. Las presentaciones elaboradas por cada } \\
\text { pareja quedarán a disposición de toda la clase. }\end{array}$} \\
\hline Act. 7 & \multirow{2}{*}{ REALIZACIÓN DE LAS PRÁCTICAS P1-P4 } & $A C-3$ \\
\hline $45 \mathrm{~min}$ & & E.V. \\
\hline \multicolumn{3}{|c|}{$\begin{array}{l}\text { El profesor pedirá a cada pareja que explique a los compañeros de forma } \\
\text { detallada cómo realizarían el análisis haciendo uso de la metodología } \\
\text { que aparece en el cuadernillo de las prácticas (material dado antes } \\
\text { del comienzo de la primera sesión). El docente intervendrá cuando sea } \\
\text { necesario para aclarar, recalcar, corregir, etc. En esta sesión se trabajará } \\
\text { tan solo con aquellas prácticas de laboratorio que, debido a la situación } \\
\text { actual de pandemia, no se van a realizar de forma presencial. En particular, } \\
\text { análisis de proteína (P1), cafeína (P2), color (P3) y humedad (P4). }\end{array}$} \\
\hline Act. 8 & \multirow{2}{*}{ REALIZACIÓN DE EJERCICIOS (P1-P4) } & $\mathrm{RE}$ \\
\hline $15 \mathrm{~min}$ & & E.V. \\
\hline
\end{tabular}

Ciclos de Mejora en el Aula (2020). Experiencias de Innovación Docente de la US Esta obra se distribuye con la licencia Creative Commons Reconocimiento-NoComercial-SinObraDerivada Internacional (CC BY-NC-ND 4.0.) 
El docente dará unos datos imaginarios para cada una de las prácticas (cómo si los alumnos las hubiesen realizado) y, tras dejar el tiempo oportuno para resolver los ejercicios, pedirá a un alumno voluntario que indique cómo los resolvería. Es decir, a partir de los datos imaginarios los alumnos deberán obtener el resultado que se busca (¿qué cantidad de proteina hay en una salchicha?, etc.)

El profesor volverá a explicar cómo se debe realizar el ejercicio para asegurar que todos los alumnos lo han comprendido.

\begin{tabular}{|c|c|c|}
\hline \multicolumn{3}{|c|}{ SESIÓN 3} \\
\hline Act. 7 & \multirow{2}{*}{$\begin{array}{c}\text { REALIZACIÓN DE LAS PRÁCTICAS } \\
\text { P5-P7 }\end{array}$} & $A C-3$ \\
\hline $2 \mathrm{~h} 30 \mathrm{~min}$ & & $\begin{array}{c}\text { Prácticas de laboratorio } \\
\text { y ficha de trabajo }\end{array}$ \\
\hline \multicolumn{3}{|c|}{$\begin{array}{l}\text { Los alumnos deberán realizar de forma individual (debido a las } \\
\text { restricciones COVID) las prácticas de análisis de almidón (P5), grasa (P6) y } \\
\text { cenizas (P7). Para ello, tendrán a disposición el cuadernillo de prácticas en } \\
\text { el que viene explicado el trabajo a realizar de forma detallada. El profesor } \\
\text { irá controlando y guiando el trabajo de los alumnos. Si se producen } \\
\text { problemas durante las prácticas (un aparato deja de funcionar, se les } \\
\text { derrama algún reactivo, etc.) el docente incentivará a los alumnos a pensar } \\
\text { en la mejor manera de resolverlo. Tras finalizar las prácticas, los alumnos } \\
\text { deberán entregar una ficha de trabajo con información sobre los datos } \\
\text { obtenidos, los problemas encontrados y las soluciones tomadas. Mediante } \\
\text { esta actividad los alumnos podrán contrastar las conclusiones a las que } \\
\text { han llegado sobre cada método analítico (sostenibilidad, dificultad, etc.). }\end{array}$} \\
\hline Act. 8 & \multirow{2}{*}{$\begin{array}{l}\text { REALIZACIÓN DE EJERCICIOS } \\
\text { (P5-P7) }\end{array}$} & $\mathrm{RE}$ \\
\hline $30 \mathrm{~min}$ & & Ficha de trabajo \\
\hline \multicolumn{3}{|c|}{$\begin{array}{l}\text { Los alumnos deberán hacer uso de los datos obtenidos en la actividad } \\
\text { anterior para conseguir el resultado que se busca (cantidad de grasa en el } \\
\text { alimento analizado, etc.). El resultado y cómo han llegado al mismo tendrán } \\
\text { que incluirlo en la ficha de trabajo. El profesor resolverá todas las dudas y } \\
\text { asegurará que todos los alumnos saben realizar los ejercicios. La ficha de } \\
\text { trabajo se entregará el día del examen. }\end{array}$} \\
\hline Act. 9 & \multirow{2}{*}{ CUESTIONARIO FINAL } & CF \\
\hline $30 \mathrm{~min}$ & & Google docs \\
\hline
\end{tabular}

Información mostrada en los recuadros: naranjas: número de la actividad (izquierda) y acrónimo (derecha); grises: duración (izquierda) y herramientas (derecha); blancos: título (superior) y descripción de la actividad (inferior). PC,

Ciclos de Mejora en el Aula (2020). Experiencias de Innovación Docente de la US Esta obra se distribuye con la licencia Creative Commons 
Preguntas Claves; $\mathrm{Cl}$, Cuestionario Inicial; IA, Idea de los Alumnos; AC, Actividad de Contraste; ER, Exposición Resultados; RE, Resolución Ejercicios; CF, Cuestionario Final; E.V., Plataforma "Enseñanza Virtual" de la Universidad de Sevilla

\section{Cuestionario inicial/final}

Como herramienta para analizar los modelos mentales de los estudiantes se hará uso de un cuestionario que los alumnos deberán resolver al principio y al final del desarrollo del CIMA (Tabla 1, Actividades 2 y 9). La evaluación de los modelos iniciales y finales de los alumnos permitirá hacer un seguimiento de la evolución de los mismos en relación con los problemas y contenidos claves y, de esta forma, obtener información muy valiosa para tomar decisiones en relación a los mapas de contenido, la metodología, las actividades y la evaluación. Este cuestionario contiene preguntas abiertas, relacionadas con problemas atractivos y relevantes y que llevan a una respuesta espontánea (Porlán, 2017). A continuación, se muestra el cuestionario inicial-final usado.

\section{¿CÓMO ANALIZAMOS LOS QUE COMEMOS?}

\section{Instrucciones:}

- Este cuestionario no tiene nota, es para ver el nivel inicial del grupo.

- Es anónimo, poner un nombre de usuario ficticio, simplemente para poder comparar los resultados en el próximo cuestionario.

- Se ruega sinceridad y no buscar información para responder en ninguna fuente (internet, libros, manuales, preguntas al compañero, etc.). Esto iría en vuestra contra pues pensaría que el nivel es más elevado del real.

- Podéis usar un lenguaje coloquial en vuestras respuestas si no encontráis un lenguaje más técnico.

Ciclos de Mejora en el Aula (2020). Experiencias de Innovación Docente de la US Esta obra se distribuye con la licencia Creative Commons 


\section{Cuestionario:}

Tienes un colega, José, que quiere montar una empresa en el sector de la alimentación porque tiene unos productos cárnicos muy ricos que quiere poner a la venta. José sabe que lo único importante en una empresa no es que los productos sepan bien y se pone en contacto contigo para que le ayudes.

1. Explicale con detalle a José por qué es importante que analice la cantidad de grasa en su producto.

2. Recomiéndale alguna metodología para ello.

3. ¿Por qué le has recomendado esa metodología y no otra? ¿En qué aspectos de los diferentes métodos te has fijado para seleccionar esta?

\section{Aplicación del CIMA}

A continuación, se comentará cómo fue el desarrollo de cada una de las actividades indicadas en la Tabla 1.

Las actividades 1 y 2 se desarrollaron sin ningún incidente y tal como estaban diseñadas. El 100\% de los alumnos rellenaron el cuestionario inicial.

Las actividades de la metodología ABP (actividades 3-6) se realizaron con unos grupos de trabajos diferentes a los diseñados previamente para poder agilizar el desarrollo de las actividades en la plataforma y por tener un número de alumnos superior al esperado. Así, se generaron tres grupos de cuatro personas y un grupo de dos personas (el trabajo a realizar se adaptó al número de personas que componían el grupo), en lugar de seis grupos de dos personas. Al reducir el número de grupos de trabajo podía ir pasando por los grupos con mayor asiduidad y resolver más fácilmente los problemas técnicos que iban surgiendo (que la conexión de los alumnos fallaba y tenía que volver a meterles en su grupo, que para enviar archivos a los Ciclos de Mejora en el Aula (2020). Experiencias de Innovación Docente de la US
Esta obra se distribuye con la licencia Creative Commons 
grupos tenía que enviarlos por correo electrónico, etc.). Por otro lado, la actividad 3 se rediseñó para realizarse en forma de debate inicial con toda la clase. El debate generado fue muy ameno y con una gran participación por parte de los estudiantes. Mi intervención fue mínima y las conclusiones a las que llegaron muy interesantes. Respondieron a las preguntas claves bastante bien, salvo la relacionada con la elección de un método de análisis. Por otra parte, me llamó la atención que nadie mencionó la obligación legal como uno de los motivos para realizar el análisis de alimentos.

Las actividades 4 y 5 se desarrollaron con una participación y una actitud muy buena por parte de los alumnos. Estos se organizaron el trabajo muy bien, pero a veces tuve que ayudar a que obtuvieran la información realmente importante de los documentos que les envíe para hacer el trabajo. Cuando finalizó la sesión casi todos los trabajos estaban prácticamente acabados, sin embargo, todos los estudiantes estuvieron aproximadamente una hora más en la plataforma mejorando el trabajo, preguntándome algunas dudas, etc.

Durante la exposición de los alumnos (actividad 6), intervine para recalcar los puntos más importantes y para que los estudiantes viesen algunos vídeos relacionados con lo que los compañeros estaban exponiendo. Al finalizar la sesión aproximadamente la mitad de la clase indicó que los vídeos les resultaron amenos, muy instructivos e interesantes. Los estudiantes se repartieron equitativamente el trabajo durante la exposición y se ciñeron al tiempo del que disponían. En general, los alumnos cumplieron con los objetivos planteados y realizaron exposiciones bastante amenas.

Ciclos de Mejora en el Aula (2020). Experiencias de Innovación Docente de la US Esta obra se distribuye con la licencia Creative Commons 
Las actividades 7 y 8 se realizaron según lo previsto. Sin embargo, algunos alumnos no pudieron hacer parte de las prácticas por un problema eléctrico y, debido a las medidas COVID, tampoco pudieron acercarse para ver cómo la realizaban sus compañeros. Estas actividades se plantearon como actividades de contraste. Así, mediante la práctica y la observación, los alumnos fueron contrastando las conclusiones a las que habían llegado el día anterior. Para incentivar este proceso fui intercalando preguntas durante la práctica. En general, se observó cierta mejoría en la realización de las prácticas en comparación con otros años.

El $100 \%$ de los alumnos respondieron al cuestionario final (actividad 9). Por otra parte, en el diseño previo se contempló preguntarles a los estudiantes qué les había parecido el CIMA de forma general y oral. Sin embargo, al final se realizó un cuestionario escrito con respuestas numéricas para poder realizar un análisis más detallado sobre la evaluación del CIMA. Así, se les pidió que valoraran (de 0 a 10) la satisfacción general con la metodología aplicada y la comprensión de los contenidos con esta metodología. También se les pidió que razonasen sus respuestas. El $100 \%$ de los alumnos respondieron a este cuestionario anónimo.

\section{Evaluación}

\section{Evaluación de los cuestionarios}

A priori, se puede deducir que los estudiantes respondieron con total sinceridad y sin buscar información a los diferentes cuestionarios. De las tres preguntas planteadas en los cuestionarios se obtuvieron tres escaleras de aprendizaje (Figura 3). El análisis global de estas escaleras nos demuestra que la metodología planteada ha sido la 
correcta para generar el conocimiento esperado. Así, se tiene que solo el $7 \%$ de las respuestas de los alumnos a las tres preguntas planteadas en el cuestionario inicial se situaron en los dos últimos peldaños de las escaleras, mientras que en el cuestionario final un mínimo del 50\% de las respuestas se situaron en estos peldaños. Otro ejemplo ilustrativo del avance conseguido en los alumnos se tiene al evaluar el último peldaño de las escaleras; en los cuestionarios iniciales ningún alumno llegó a este nivel, mientras que al final entre el 43 y el $50 \%$ de los alumnos llegaron a este nivel. A continuación, se evaluarán cada una de las escaleras de aprendizaje de forma individual.

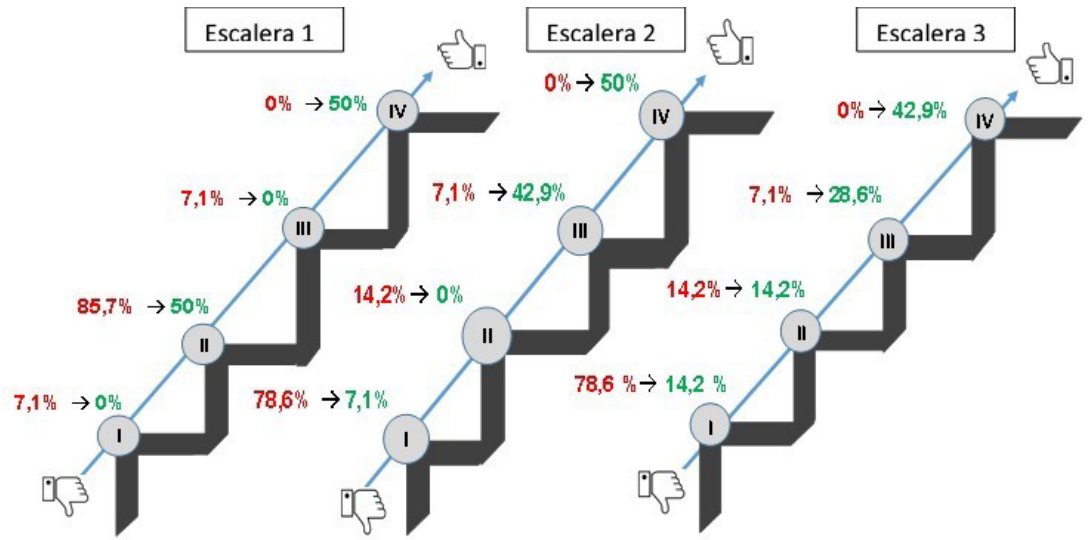

Figura 3. Escalera de aprendizaje de la pregunta 1 (izquierda), 2 (en medio) y 3 (derecha).

En rojo respuestas en el cuestionario inicial y en verde en el final. I-IV diferentes niveles de respuesta

En la escalera 1 se representan las respuestas a la pregunta ¿Por qué es importante evaluar la grasa? Las respuestas han sido clasificadas en cuatro niveles: I. No responde o responde sin sentido, II. Da motivos relacionados con la salud (para que el consumidor sepa la cantidad de grasa que ingiere y para que el vendedor pueda vender productos saludables), III. Indica otros motivos y los explica vagamente y IV. Indica varios motivos y los defiende

Ciclos de Mejora en el Aula (2020). Experiencias de Innovación Docente de la US Esta obra se distribuye con la licencia Creative Commons 
con lógica. Del análisis de las respuestas a esta pregunta destaco el hecho de que una parte importante de los estudiantes ha conseguido entender los aspectos legales relacionados con el etiquetado de los alimentos, lo cual está relacionado con otros contenidos que se ven en los seminarios de esta asignatura. Se ha conseguido así dar cierta conexión entre las distintas partes de esta asignatura. En esta escalera encuentro principalmente un obstáculo que explica la mayor altura de los dos últimos escalones de esta escalera. Este obstáculo está relacionado con el hecho de que a los estudiantes les cuesta profundizar en sus pensamientos para llegar a conclusiones más allá de las relacionados con las ideas previas que tienen muy arraigadas. Así, la mayoría de los alumnos tienen muy clara la relación consumo de grasa-salud y se han enfocado en ello para responder a la pregunta, dejando de lado otras respuestas interesantes que, de haber pensado un poco más, podrían haber encontrado.

Por otro lado, en la escalera 2 se clasifican las respuestas a la pregunta ¿Qué metodología recomendarías?: I. No responde o responde sin sentido, II. No conoce la metodología, pero aplica la lógica, III. Indica correctamente la metodología y IV. Explica correctamente la metodología y/o indica varias posibles. Esta escalera nos demuestra cómo se ha conseguido que muchos alumnos conozcan las posibles metodologías a usar, entiendan sus principios y sepan que no sólo existe una posible. Uno de los principales obstáculos que encuentro en esta escalera es la dificultad que tienen los estudiantes para aplicar la lógica. Ellos saben o no saben el nombre de la metodología, pero en el caso de no saber el nombre de la misma no son capaces de razonar para concluir qué principios básicos debería tener dicha metodología atendiendo a lo que se está analizando. Otra dificultad encontrada está relacionada con la explicación de la metodología. El hecho de que algo menos de la mitad de la clase se situara en el penúltimo peldaño y

Ciclos de Mejora en el Aula (2020). Experiencias de Innovación Docente de la US Esta obra se distribuye con la licencia Creative Commons 
no alcanzara el último lo achaco, principalmente, al hecho de que muchos alumnos no pudieron realizar esta práctica por problemas técnicos. Al no realizar la práctica no la entendieron del todo bien. Además, también puede deberse a que en el cuestionario no quedaba del todo claro que, además de dar el nombre de la metodología, tenían que explicarla.

Por último, la escalera 3 representa la clasificación a la pregunta ¿Por qué has seleccionado esta metodología?: I. No responde o responde sin sentido, II. Indica que es una metodología apropiada sin dar muchas explicaciones, III. Indica ciertas características de la metodología sin dar explicaciones y IV. Indica bastantes características del método y las razona. De la evaluación de las respuestas a esta pregunta destaco el hecho de que se ha conseguido que un buen número de alumnos entienda la importancia de ciertos aspectos actitudinales a la hora de seleccionar un método de análisis. Así, muchos estudiantes han indicado que el método Soxhlet es un método respetuoso con el medio ambiente y barato. Estos contenidos actitudinales se han incorporado por primera vez en la asignatura al aplicar este CIMA. En el cuestionario inicial, la gran mayoría de los estudiantes (79\%) no respondieron a esta pregunta ya que, cómo indicaron, no conocían ninguna metodología. Es decir, no tenían conocimientos previos suficientes para poder responder. Tras la aplicación del CIMA los alumnos pudieron enfrentarse a esta pregunta con conocimientos suficientes. Sin embargo, un 14\% de los alumnos aplicaron para responderla un pensamiento que, aunque era lógico, era demasiado sencillo (nivel II): si he usado esta metodología será porque es útil. Se tiene así el mayor obstáculo en esta escalera, la dificultad de aplicar pensamientos más complejos que den lugar a respuestas más desarrolladas y completas. Este obstáculo es el que dificulta el avance desde el nivel II al III y del III al IV. Pero el obstáculo más grande se encuentra entre el penúltimo y último escalón.

Ciclos de Mejora en el Aula (2020). Experiencias de Innovación Docente de la US Esta obra se distribuye con la licencia Creative Commons 
Para responder correctamente, los alumnos deben tener conocimientos previos, aplicarlos al caso concreto, pensar en una explicación y desarrollarla con claridad. Atendiendo a la dificultad para llegar a este nivel considero que el CIMA se ha desarrollado con cierto éxito ya que casi la mitad de la clase ha llegado a este nivel.

\section{Evaluación del CIMA}

En general, el CIMA ha sido una experiencia muy gratificante y, en mi opinión, el desarrollo del mismo ha sido bastante fructífero. La participación, motivación e interés de los estudiantes ha sido uno de los puntos más positivos de este ciclo de mejora. Además, como se deduce de la evaluación de los cuestionarios y de la calificación de los exámenes (resultados no mostrados), la mayoría de los estudiantes ha alcanzado los objetivos académicos planteados. Destaco el hecho de que se han conseguido incorporar contenidos actitudinales no contemplados previamente en la asignatura. Del cuestionario relativo a la metodología se deduce que, en general, los alumnos están bastante satisfechos con el desarrollo del CIMA. Así la valoración media a la metodología aplicada ha sido de 7,8, mientras que al nivel de comprensión de los contenidos adquirido con esta metodología se le ha dado un valor medio de 7,1. Entre los razonamientos de los estudiantes a estas respuestas extraigo como conclusión que les ha parecido útil y motivante el hecho de que sean ellos los protagonistas de su aprendizaje y el uso de vídeos, si bien alguno ha criticado el uso de vídeos en inglés. Por otro lado, el desarrollo de este CIMA ha permitido que los alumnos tengan un material extra para estudiar, ya que los trabajos elaborados se pusieron a disposición del resto de compañeros.

Por otro lado, la adaptación de las actividades al tiempo estipulado es uno de los aspectos que creo deberían ser mejorados. Para ello, entre otras medidas, se deberían dar unas pautas más detalladas a los alumnos a la hora de Ciclos de Mejora en el Aula (2020). Experiencias de Innovación Docente de la US
Esta obra se distribuye con la licencia Creative Commons 
realizar el trabajo para que sepan buscar la información más relevante entre los documentos que se envíen. Otro aspecto a mejorar es el cuestionario, ya que creo que en algunos casos los alumnos no han entendido lo que se les preguntaba. Por ello, sería necesario utilizar preguntas más claras y acompañarlas de una explicación previa para asegurar que todo el mundo comprende lo que se pregunta. Por último, durante el desarrollo del CIMA, he podido comprobar que los alumnos han interiorizado mucho mejor los contenidos relacionados con el trabajo realizado por ellos mismos que aquellos que habían sido explicados por los compañeros con ayuda del profesor. Por ello, creo que sería interesante que todos los grupos trabajen exclusivamente con la temática seleccionada previamente como la más importante, en lugar de que cada grupo trabaje con una temática diferente. De esta forma, se podría conseguir que toda la clase aprenda, como mínimo, los contenidos más importantes.

\section{Principios Didácticos de referencia}

Entre los principios didácticos fundamentales que han guiado esta experiencia destaco el uso del trabajo de los alumnos como pilar fundamental de su aprendizaje ya que considero que así se consigue un aprendizaje más profundo y duradero a la vez que se consigue motivar al estudiante, ya que este pasa a tener un papel más activo durante las clases. Es por ello que, en un futuro, me gustaría mantener este principio docente.

Además, considero un principio didáctico de referencia el controlar, durante el desarrollo de las sesiones, la evolución y el aprendizaje de los alumnos, pues el principal objetivo de la labor docente es que los estudiantes aprendan. Para ello, creo que se deben plantear cuestiones frecuentemente en clase para, a través de las respuestas de

Ciclos de Mejora en el Aula (2020). Experiencias de Innovación Docente de la US Esta obra se distribuye con la licencia Creative Commons 
los estudiantes, sacar conclusiones sobre el avance de los mismos. Se puede así ir adaptando las sesiones y se evita que se comiencen a tratar los contenidos más complejos sin que los alumnos hayan entendido los contenidos más sencillos. Considero por tanto importante también preguntar asiduamente a los alumnos si han entendido las explicaciones y analizar cuáles son los principales obstáculos en el avance.

Para poder controlar la evolución y el aprendizaje de los alumnos es necesario que estos no tengan miedo a las repercusiones que puede tener responder mal a una pregunta, dar opiniones, preguntar algo demasiado básico, etc. Por ello, es fundamental crear un ambiente adecuado para eliminar estos miedos. Para ello, creo que el docente debe ser cercano, tratar siempre con respeto al alumnado, incentivar y motivar su curiosidad y fomentar la comunicación bidireccional.

Por último, el tener las clases diseñadas meticulosamente previamente al desarrollo de las mismas ha sido clave para conseguir unos resultados tan satisfactorios, por lo que intentaré en el futuro incorporar esta actividad de forma habitual en mi labor docente.

Ciclos de Mejora en el Aula (2020). Experiencias de Innovación Docente de la US Esta obra se distribuye con la licencia Creative Commons 
Palabras clave: Prácticas de Nutrición y Bromatología, Grado en Farmacia, Docencia Universitaria, Experimentación Docente Universitaria.

Keywords: Practical subject Nutrition and Bromatology, Pharmacy Degree, University Teaching, University Teaching Experimentations.

\section{Referencias bibliográficas}

Bain, K. (2007). Lo que hacer los mejores profesores universitarios. Valencia: Publicacions de la Universitat de Valéncia.

Cubero, R. y Carmona, M. J. (1994). Construcción del Conocimiento en el Aula: Procesos de Aprendizaje. En: Servicio de Publicaciones del I.C.E. de la Universidad. C.A.P. Materiales Didácticos: Didáctica General y Psicología de la Educación (pp. 107-129). Sevilla: Instituto de Ciencias de la Educación de la Universidad de Sevilla.

Finkel, D. (2008). Dar clase con la boca cerrada. Valencia: Publicacions de la Universitat de Valéncia.

Porlán, R. (Coord.) (2017). Enseñanza Universitaria. Cómo mejorarla. Madrid: Morata.

Ciclos de Mejora en el Aula (2020). Experiencias de Innovación Docente de la US Esta obra se distribuye con la licencia Creative Commons 